\title{
Voriconazole-refractory invasive aspergillosis
}

\author{
Se Yoon Park, Jung-A Yoon, and Sung-Han Kim
}

Department of Infectious Diseases, Asan Medical Center, University of Ulsan College of Medicine, Seoul, Korea

Received: January 13, 2017

Accepted: August 6, 2017

\section{Correspondence to}

Sung-Han Kim, M.D.

Department of Infectious

Diseases, Asan Medical Center,

University of Ulsan College of

Medicine, 88 Olympic-ro 43-gil,

Songpa-gu, Seoul 05505, Korea

Tel: $+82-2-3010-3305$

Fax: $+82-2-3010-6970$

E-mail: kimsunghanmd@hotmail.com
Invasive aspergillosis (IA) is one of the most common life-threatening complications in immunocompromised patients. Voriconazole is currently the drug of choice for IA treatment. However, some patients with IA suffer clinical deterioration despite voriconazole therapy. Management of voriconazole-refractory IA remains challenging; no useful recommendations have yet been made. Voriconazole-refractory IA can be further categorized as disease attributable to misdiagnosis or co-infection with another mold; inadequate blood voriconazole blood; inadequate tissue drug concentrations attributable to angioinvasion; immune reconstitution inflammatory syndrome; or infection with voriconazole-resistant Aspergillus. Hence, when encountering a case of voriconazole-refractory IA, it is necessary to schedule sequential tests to decide whether medical treatment or surgical intervention is appropriate; to adjust the voriconazole dose via drug monitoring; to seek $\mathrm{CYP}_{2} \mathrm{C}_{19}$ polymorphisms; to monitor serum galactomannan levels; and to examine the drug susceptibility of the causative Aspergillus species.

Keywords: Aspergillosis; Voriconazole; Therapy; Aspergillus; Immunocompromised host

\section{INTRODUCTION}

Invasive aspergillosis (IA), especially invasive pulmonary aspergillosis (IPA), is an opportunistic infection developing principally in patients with neutropenia, and in recipients of hematopoietic stem cells and solid organ transplants $[1,2]$. In the time since a landmark study [3] of primary IA therapy showed that the mortality rate in a voriconazole-treated group (29\%) was lower than that in an amphotericin-B-treated group (42\%), voriconazole has been the drug of choice for IA treatment [4]. However, some patients with IA exhibit clinical deterioration despite voriconazole therapy. Management of voriconazole-refractory IA remains challenging; no useful recommendations have yet been made. Voriconazole-refractory IA can be further categorized as disease attributable to (1) misdiagnosis or co-infection with another mold; (2) inadequate voriconazole blood levels; (3) inadequate drug concentrations in tis- sue attributable to angioinvasion; (4) immune reconstitution inflammatory syndrome (IRIS); or (5) infection with voriconazole-resistant Aspergillus. In this review, we discuss case examples of each category, and offer guidance as to how such difficult clinical situations may be managed.

\section{MISDIAGNOSIS OR COINFECTION WITH AN- OTHER MOLD}

\section{Pitfalls of non-culture-based diagnosis}

A definitive IA diagnosis is usually based on the isolation and identification of an Aspergillus species and/ or microscopic evidence of fungi in histopathological specimens [5]. However, the low sensitivity of fungus culture from clinical specimens (10\% to $30 \%$ [6]) and the need for (invasive) biopsies in critically ill patients limit the occasions when definitive diagnoses are possible. 
Recently, the criteria of the European Organization for Research and Treatment of Cancer/Invasive Fungal Infections Cooperative Group (EORTC) have been widely used to diagnose IA, although these criteria are not recommended for use in daily clinical practice [7]. Caution is needed when diagnosing probable IA on the basis of positive galactomannan (GM) test results; the assay lacks both sensitivity and specificity. Numerous instances of false-positive and false-negative GM test results have been reported in patients with suspected IA [8-10]. The following case is a typical example. A 6o-year female received an ABO-incompatible kidney transplant. Acute rejection developed soon after transplantation, resulting in graft failure. Four months after transplantation, the patient presented with fever, and a new lung nodule was detected on chest X-ray. Chest computed tomography (CT) revealed a mass-like consolidation (Fig. 1A). The bronchoalveolar lavage (BAL) fluid was positive on the GM assay, although the serum was negative. No other pathogen was isolated and the final mycobacterial cul- ture of BAL fluid (performed 8 weeks later) was negative. She was clinically diagnosed with probable IPA (using the EORTC criteria) and received voriconazole therapy. However, the chest mass-like consolidation increased despite 16 weeks of therapy, and many supraclavicular and mediastinal lymph nodes became enlarged (Fig. 1B). The supraclavicular lymph node and a lung were both biopsied. The specimens exhibited chronic granulomatous inflammation with caseous necrosis. Voriconazole therapy was discontinued and empirical anti-tuberculosis (TB) therapy commenced. Six weeks later, a member of the Mycobacterium tuberculosis complex was cultured from sputum and aspirated lung tissue, and the masslike consolidation in the right lung became reduced in size after 8 weeks of anti-tuberculous therapy (Fig. 1C and ${ }_{1 D}$ ). Thus, in this case, TB had been misdiagnosed as IPA because TB may occasionally present with atypical clinical manifestations in immunocompromised patients. Therefore, T'B mimicking IPA should be considered in TB-endemic areas (such as South Korea). We
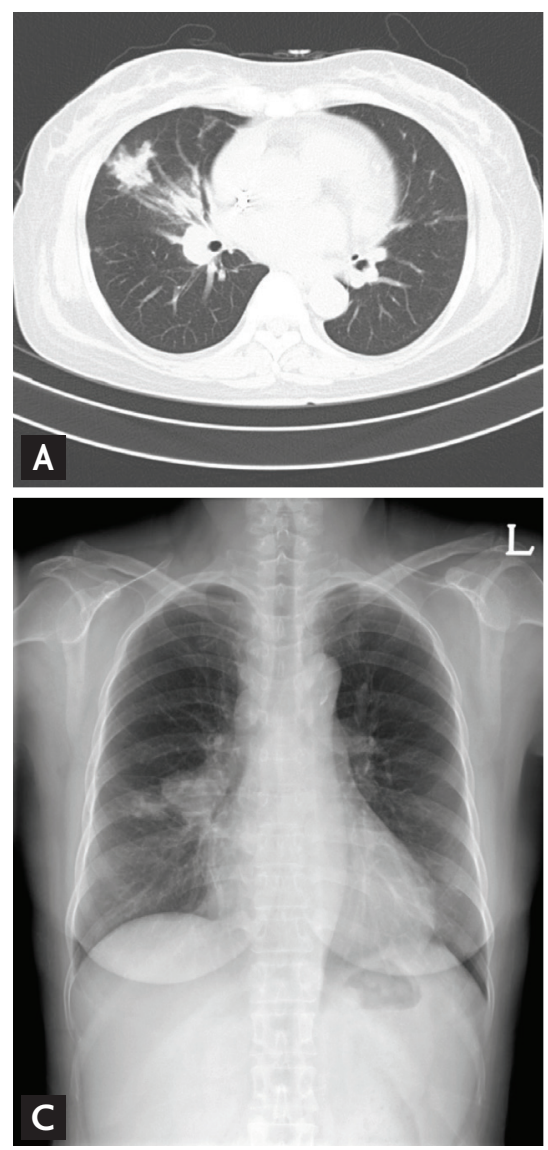
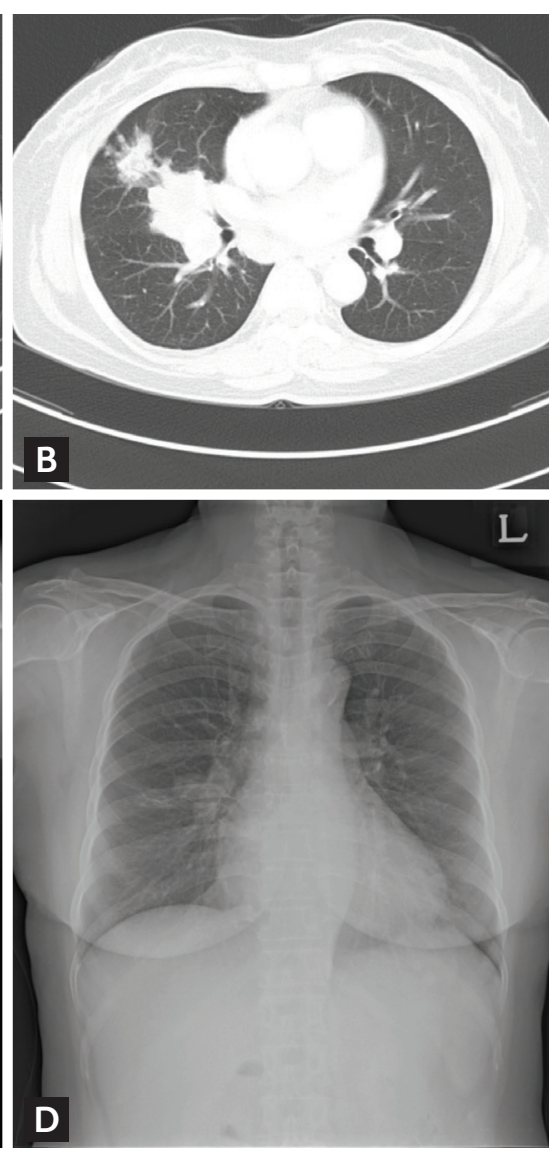

Figure 1. Images obtained from a 6o-year-old female with tuberculosis mimicking invasive aspergillosis. She had received a kidney transplant 4 months prior. (A, B) conventional computed tomography (CT) lung images (5-mm-thick) obtained at the level of the right middle lobe (RML). (A) Image reveals macronodular consolidation along the bronchovascular bundle of the RML, and bronchial wall thickening. The bronchoalveolar lavage fluid was strongly positive for galactomannan and the initial diagnosis was of probable invasive pulmonary aspergillosis. (B) After 4 months of voriconazole treatment, follow-up chest CT showed that the irregularly shaped mass had increased in size and exhibited a tree-inbud appearance. Percutaneous core lung and supraclavicular lymph node biopsies revealed chronic granulomatous inflammation with caseous necrosis. A member of the Mycobacterium tuberculosis complex was cultured from sputum and aspirated lung tissue. The patient received empirical anti-tuberculous therapy. Follow-up chest radiographs showed that the RML lung nodules had decreased in size (C: before anti-tuberculous therapy; D: after 8 weeks of anti-tuberculous therapy). 
recently found that about one-thirds of transplant recipients with TB underwent initial antifungal therapy and $20 \%$ to $30 \%$ showed positive GM test results [11]. Thus, misdiagnosis should be considered in voriconazole-unresponsive patients in whom IA is indicated based on non-culture-based assays, such as the GM test. It is likely that some patients diagnosed with probable IPA based on positive GM test data have in fact been misdiagnosed and treated with voriconazole in the past, with adverse outcomes. It is worth emphasizing that isolation of an Aspergillus species from even a non-sterile specimen yields important information in terms of IA status. The EORTC criteria state that a positive non-sterile culture result, combined with a positive GM assay outcome, afford the same level of evidence as a single positive mycological factor. Notably, we found that all five probable IPA cases with positive non-sterile culture results were also positive in the aspergillosis immunohistochemistry (IHC) assay only. Of 12 probable IPA cases for whom the GM assays were positive, only five (42\%) were positive on the aspergillosis IHC test [6]. We thus infer that non-sterile culture results are more accurate than non-culture-based tests, such as the GM assay. Hence, we propose that the EORTC criteria should be stratified further into probable IPA based on GM test results and highly probable IPA based on non-sterile culture results.

\section{Limitations of histomorphological diagnoses}

Histomorphological diagnoses of invasive mold infections are usually definitive. However, some limitations should be borne in mind. The following is an extreme example. A 62-year female received a kidney transplant. On postoperative day 8 , she developed severe abdominal pain and a sigmoid perforation with panperitonitis, and underwent total colectomy. The pathology of the resected colon (Fig. 2A) was morphologically compatible with mucormycosis (Fig. 2B). She therefore received conventional, amphotericin-B antifungal therapy. Despite 10 days of such therapy, she deteriorated clinically and a second-look operation was performed. Total gastrectomy, splenectomy, graftectomy, and ileal repair were required because the uncontrolled infection had extended to the liver, spleen, stomach, and small bowel. The fungal hyphae seen in the stomach, liver, and spleen branched at an acute angle and were septated, suggestive of aspergillosis rather than mucormycosis (Fig. 2C). The initial
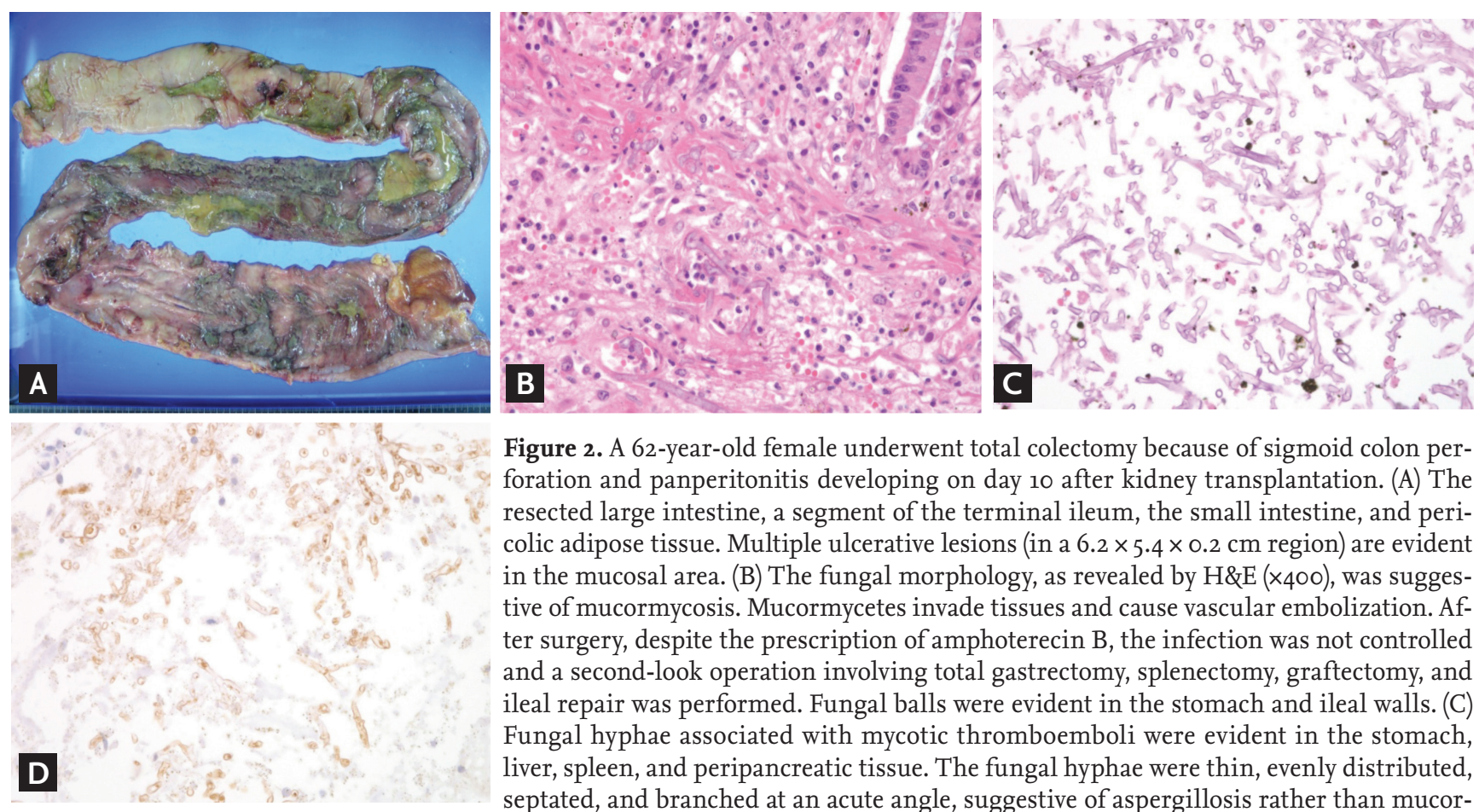

Figure 2. A 62-year-old female underwent total colectomy because of sigmoid colon perforation and panperitonitis developing on day 10 after kidney transplantation. (A) The resected large intestine, a segment of the terminal ileum, the small intestine, and pericolic adipose tissue. Multiple ulcerative lesions (in a $6.2 \times 5.4 \times 0.2 \mathrm{~cm}$ region) are evident in the mucosal area. (B) The fungal morphology, as revealed by H\&E ( $\times 400)$, was suggestive of mucormycosis. Mucormycetes invade tissues and cause vascular embolization. After surgery, despite the prescription of amphoterecin B, the infection was not controlled and a second-look operation involving total gastrectomy, splenectomy, graftectomy, and ileal repair was performed. Fungal balls were evident in the stomach and ileal walls. (C) Fungal hyphae associated with mycotic thromboemboli were evident in the stomach, liver, spleen, and peripancreatic tissue. The fungal hyphae were thin, evenly distributed, septated, and branched at an acute angle, suggestive of aspergillosis rather than mucormycosis (periodic acid-Schiff staining, $\times 400$ ). (D) Mucormycosis immunohistochemistry (IHC) staining $(\times 400)$ was positive and aspergillosis IHC was negative. 
pathology was reviewed and the final pathological report was revised from a morphological diagnosis compatible with mucormycosis to a diagnosis compatible with aspergillosis. The patient's clinical condition deteriorated and she died 6 days after the second-look operation. The aspergillosis IHC test was negative but the mucormycosis IHC test was positive (Fig. 2D). Thus, the morphology of an infecting fungus may be confusing, indicating that morphological diagnosis has certain limitations.

We recently found that the extent of overall agreement in terms of histomorphological diagnoses made by two experienced pathologists was $87 \%$ [6]. Hence, disagreements were evident in $13 \%$ of cases. In addition, we earlier showed that $25 \%$ of pulmonary aspergillosis cases diagnosed based on both fungal morphology and other mycological evidence of aspergillosis were in fact incorrectly diagnosed (pulmonary mucormycosis had been interpreted as pulmonary aspergillosis) [6]. Therefore, the morphology of the infecting fungus alone is of limited utility in the diagnosis of aspergillosis.

\section{Coinfection or colonization by other molds}

Coinfection should be considered in cases of voriconazole -refractory IPA. An earlier study found that respiratory cultures of 13 of 17 patients ( $76 \%$ ) with definite or probable pulmonary mucormycosis were also positive for another pathogen, and cultures of six of 13 histologically-proven cases $(46 \%)$ of pulmonary mucormycosis yielded other molds, including three Aspergillus species and two Fusarium species [12]. We also earlier showed that positive GM test results were not uncommon in patients with proven or probable mucormycosis [6].

We do not know whether other mold infections revealed by a positive GM test result, or culture of another mold, in patients with pulmonary mucormycosis are attributable simply to opportunistic colonization of damaged bronchi or true tissue-invasive coinfection. In addition, we do not know if frequent coinfection with another fungus in mucormycosis patients is exclusive to such patients, and thus absent in patients with aspergillosis; few studies concerning this have appeared. However, it is necessary to be cautious when interpreting non-sterile culture results and non-culture-based assays in patients with voriconazole-refractory IA; coinfection with another mold is a possible differential diagnosis. Combined antifungal therapy (voriconazole plus anidulafungin [13] or liposomal amphotericin plus caspofungin $[14,15])$, sequential antifungal therapy (voriconazole followed by liposomal amphotericin [16]), and prescription of broader-spectrum azole agents such as isavuconazole [17] and posaconazole [18] may all be valuable in such uncertain situations.

\section{INADEQUATE VORICONAZOLE BLOOD LEVELS}

Many previous studies have shown that inadequate voriconazole blood trough levels are associated with treatment failure in patients with IPA [19,20], although some studies, including ours [21], failed to detect an association between the blood voriconazole level and clinical outcomes. A recent randomized trial showed that therapeutic drug monitoring in patients undergoing voriconazole therapy improved the treatment responses [22]. Intuitively, it might seem that the voriconazole level should influence outcomes. However, the optimal timing of blood trough level sampling, yielding accurate voriconazole concentrations, remains unclear, as does the optimal dose level. Inter- and intra-dose voriconazole trough levels vary [19-21], probably attributable to unrecognized confounders, including variations in intake, comedication levels, renal and liver disease, and polymorphisms in the gene encoding cytochrome $\mathrm{CYP}_{2} \mathrm{C}_{19}$ [21]. A particular CYP2C19 polymorphism triggering rapid voriconazole metabolism has been associated with treatment failure [23]. Thus, $C Y$ $\mathrm{P}_{2} \mathrm{C}_{19}$ polymorphisms should be explored in patients with voriconazole-refractory IA. CYP2 $\mathrm{C}_{19}$ mutations encoding aberrant isoenzymes that function poorly are more common in Asians than non-Asians (60\% to $70 \%$ of Asians vs. $30 \%$ of Caucasians and Afro-Africans). Thus, ensuring that the voriconazole blood levels are adequate, adjusting the dose if required, and evaluating $\mathrm{CrP}_{2} \mathrm{C}_{1} 9$ polymorphism status are essential when treating patients with voriconazole-refractory IA.

\section{INADEQUATE DRUG TISSUE CONCENTRA- TIONS ATTRIBUTABLE TO ANGIOINVASION}

Angioinvasion is a feature of IA [24]. If blood vessels at the target site are damaged, the drug cannot reach the target site although the blood level appears to be ade- 
quate. A typical example follows. A 50-year-old female underwent chemotherapy to treat acute myeloid leukemia. Febrile neutropenia developed early. Chest CT revealed irregular consolidation accompanied by ground glass opacity and the halo sign (Fig. $3 \mathrm{~A}$ and ${ }_{3} \mathrm{C}$ ). Serum GM assays were strongly positive. In line with the EORTC criteria, the patient was diagnosed with probable IPA and received intravenous, followed by oral, voriconazole, the serum levels of which ( 3 to $4 \mathrm{mg} / \mathrm{dL}$ ) seemed to be appropriate. One week after discharge, she presented to our emergency department with massive hemoptysis, and was intubated to protect the airway. She continued on voriconazole (200 mg twice daily); the trough blood level was $2.17 \mathrm{mg} / \mathrm{dL}$. Follow-up chest CT revealed aspirated blood in the right middle and right lower lobes (Fig. 3B and 3D). She underwent emergency surgery to treat the massive hemoptysis. Pathological evaluation of the resected lung specimen revealed IA involving the pulmonary artery (see insets in Fig. $3 \mathrm{~B}$ and ${ }_{3} \mathrm{D}$ ). She recovered with no surgical complications; voriconazole therapy was maintained for about 6 months. We infer that switching to another antifungal might not have been helpful. The main pulmonary ar- tery had been invaded and no systemically administered drug could reach the target site (i.e., the pulmonary artery). Therefore, a thorough evaluation of angio-invasive status, followed by surgical intervention (if necessary), is essential in some patients with voriconazole-refractory IA; switching to another antifungal agent is not so helpful in this clinical situation.

\section{IMMUNE RECONSTITUTION INFLAMMATORY SYNDROME}

Both clinical and radiological deterioration is sometimes observed during neutrophil recovery in IA patients; those with progressive IA and IRIS should be distinguished in this context. The median lesion volume in neutropenic patients with IPA, measured serially via CT, increased 4-fold from day o to day 7 despite antifungal therapy [25]. However, many physicians consider that radiological deterioration in IPA patients during neutrophil recovery is associated with progression of infection, triggering modifications of antifungal therapy, with or without surgery [26]. The GM titer may indeed reflect
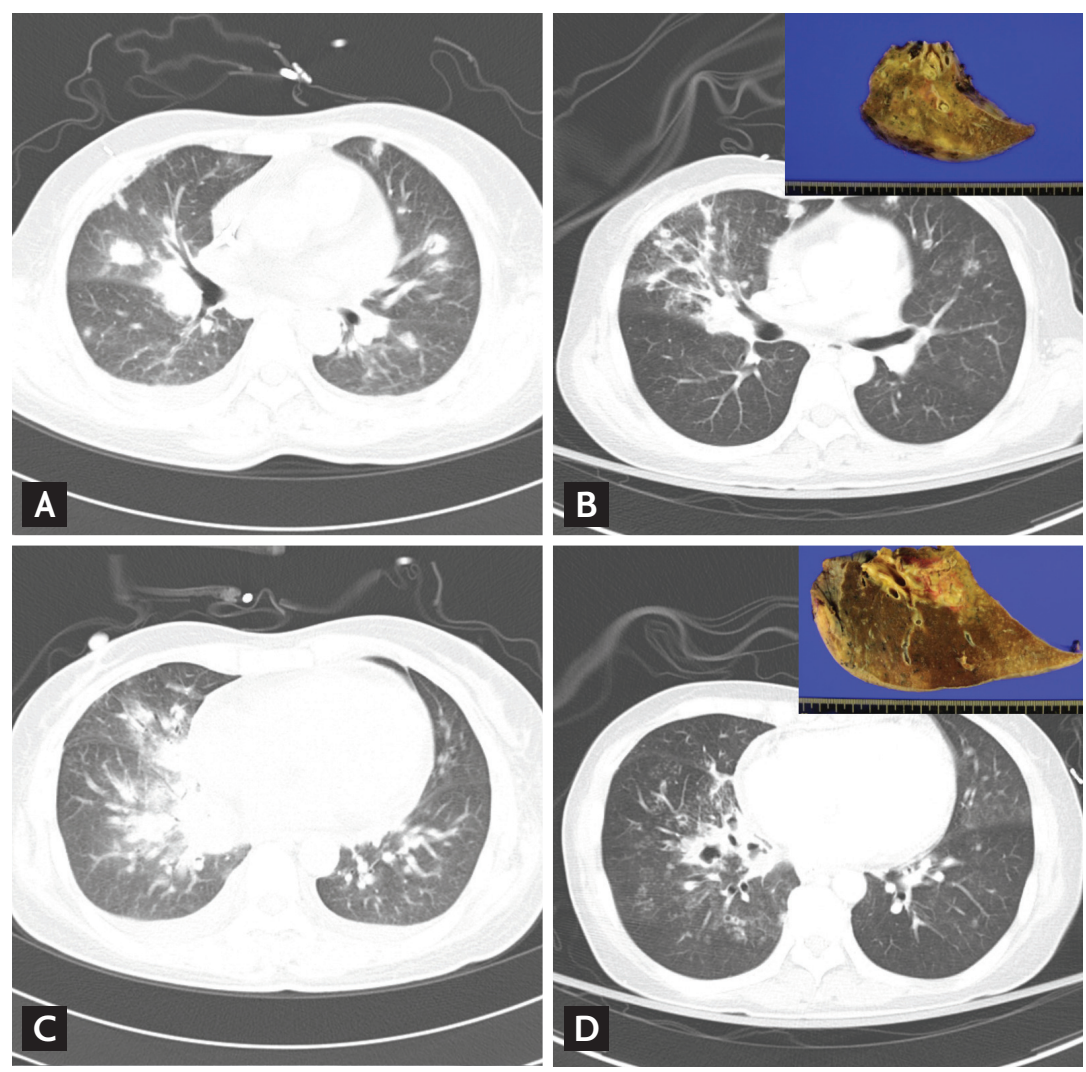

Figure 3. Computed tomography (CT) images obtained from a 50-year-old female with angio-invasive pulmonary aspergillosis under induction chemotherapy. (A, B) High-resolution CT lung images (1-mm-thick) obtained at the level of the right middle lobe. Ill-defined macronodules with halo signs are evident in both lungs. One week after discharge, the patient presented to the emergency department with massive hemoptysis. (C, D) Conventional CT images (5-mm-thick) revealed that the number and size of nodules had increased in both lungs (especially the right lung), and cavitary changes were evident. The patient underwent bilobectomy of the right middle and right lower lobes. The pathology of the resected lung revealed aspergillosis featuring pulmonary artery invasion (insets in B and D). 


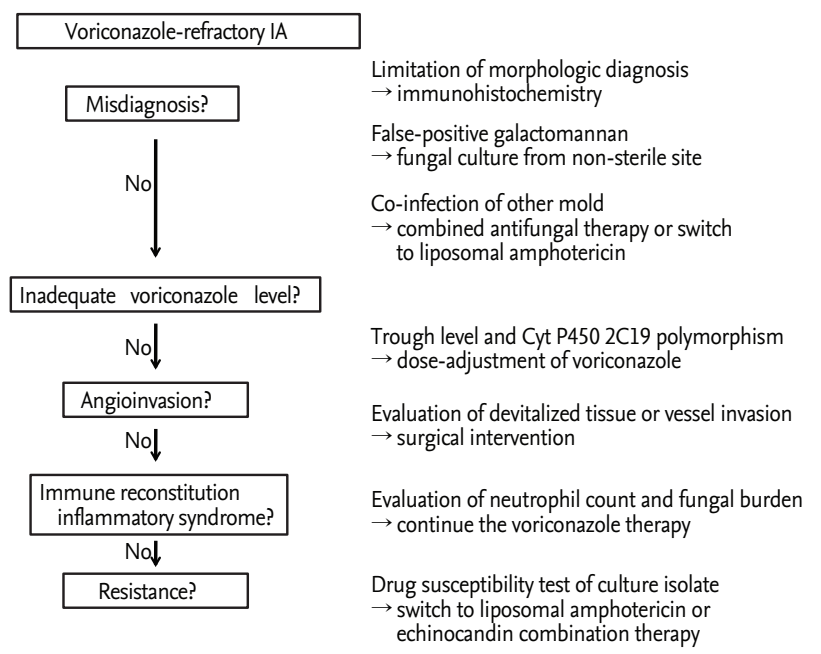

Figure 4. A proposed sequential approach to investigating cases of voriconazole-refractory aspergillosis.

the fungal burden. However, it may be possible to determine whether radiological deterioration is attributable to either IPA progression or IRIS by evaluation of clinical and microbiological parameters, and immunological measures including neutrophil counts. We recently showed that IRIS developed in $25 \%$ of neutropenic patients with IPA, and in about $75 \%$ of such patients exhibiting neutrophil recovery from radiologically progressive disease [27]. Notably, for an unknown reason, voriconazole seems to trigger IRIS more often than do other antifungal agents [27]. Some antifungal agents exert immunomodulatory effects [28]. The increasing number of available antifungals encourages physicians to change or combine these agents when treating patients exhibiting deteriorating radiological findings during neutrophil recovery. About $66 \%$ of our patients had IRIS, associated with reduced mortality and prognoses as good as those of patients exhibiting favorable radiological responses [27]. We therefore believe that close monitoring, without modification of antifungal treatment, is warranted in such patients.

\section{VORICONAZOLE-RESISTANT ASPERGILLUS}

Although some Aspergillus species are naturally resistant to azoles, the emergence of acquired azole-resistance has reduced the use of azole drugs in clinical practice [29].
Acquired azole-resistance may be attributable to longterm exposure of patients to such drugs or environmental exposure to azole compounds [30]. Horizontal gene transfer, commonly associated with the spread of bacterial resistance, is uncommon in fungi; azole-resistance may be acquired by patients (such as those with aspergillosis) undergoing long-term azole treatment [30]. However, environmentally derived resistance is possible when patient risk factors are not evident, particularly in geographical regions where azole fungicides are used to protect crops [30]. Review of local epidemiological data is essential in this context.

RecentUKand Dutch studies reported itraconazole-resistance frequencies of 5.0\% and 5.3\%, respectively; the levels of such resistance have recently increased markedly $[31,32]$. In contrast, a Spanish study found that the frequency of itraconazole resistance was only 1.3\% [33]. Unfortunately, few studies have yet addressed this issue in South Korea. However, we assume that azole-resistant Aspergillus spp. may be uncommon in South Korea because such strains are rare in Japan [34].

Although no controlled trial has yet compared recovery from azole-resistant and azole-susceptible infections, preclinical experimental models indicate that azole resistance reduces the efficacy of azole monotherapy [35], and many clinical studies have reported that azole resistance was associated with treatment failure [31,32,35,36]. No guidelines are yet available for the management of azole-resistant IA, but most experts recommend switching from voriconazole to liposomal amphotericin $\mathrm{B}$, or a voriconazole/echinocandin combination [30].

\section{PROPOSED APPROACH TO TREATMENT OF VORICONAZOLE-REFRACTORY IA}

Although early diagnosis and prompt initiation of voriconazole treatment improves the survival rate of patients with IA [37], we have occasionally encountered voriconazole-refractory aspergillosis cases. A schematic summarizing our proposed approach to the treatment of voriconazole-resistant IA in South Korea is shown in Fig. 4; such a sequential approach may help us to understand the mechanistic basis of resistance, and effectively manage individual cases of voriconazole-refractory IPA. 


\section{CONCLUSIONS}

In conclusions, our proposed categorization including (1) misdiagnosis or coinfection, (2) inadequate voriconazole blood levels, (3) inadequate drug concentration in target tissue due to angioinvasion, (4) IRIS, and (5) voriconazole-resistant aspergillosis will help physicians deal with this difficult clinical situation, when encountering a case of voriconazole-refractory IA.

\section{Conflict of interest}

No potential conflict of interest relevant to this article was reported.

\section{Acknowledgments}

This study was supported by a grant from the Korea Health Technology R\&D Project via the Korea Health Industry Development Institute (KHIDI), funded by the Ministry of Health and Welfare, Republic of Korea (no. HI16Co272).

\section{REFERENCES}

1. Kim SH, Moon SM, Han SH, et al. Epidemiology and Clinical outcomes of invasive pulmonary aspergillosis: a nationwide multicenter study in Korea. Infect Chemother 2012;44:282-288.

2. Yun JH, Lee SO, Jo KW, et al. Infections after lung transplantation: time of occurrence, sites, and microbiologic etiologies. Korean J Intern Med 2015;30:506-514.

3. Herbrecht R, Denning DW, Patterson TF, et al. Voriconazole versus amphotericin $\mathrm{B}$ for primary therapy of invasive aspergillosis. N Engl J Med 2002;347:408-415.

4. Walsh TJ, Anaissie EJ, Denning DW, et al. Treatment of aspergillosis: clinical practice guidelines of the Infectious Diseases Society of America. Clin Infect Dis 2008;46:327360.

5. Hope WW, Walsh TJ, Denning DW. Laboratory diagnosis of invasive aspergillosis. Lancet Infect Dis 2005;5:609-622.

6. Jung J, Park YS, Sung H, et al. Using immunohistochemistry to assess the accuracy of histomorphologic diagnosis of aspergillosis and mucormycosis. Clin Infect Dis 2015;61:1664-1670.

7. De Pauw B, Walsh TJ, Donnelly JP, et al. Revised definitions of invasive fungal disease from the European Orga- nization for Research and Treatment of Cancer/Invasive Fungal Infections Cooperative Group and the National Institute of Allergy and Infectious Diseases Mycoses Study Group (EORTC/MSG) Consensus Group. Clin Infect Dis 2008;46:1813-1821.

8. Kedzierska A, Kochan P, Pietrzyk A, Kedzierska J. Current status of fungal cell wall components in the immunodiagnostics of invasive fungal infections in humans: galactomannan, mannan and $(1 \rightarrow 3)$-beta-D-glucan antigens. Eur J Clin Microbiol Infect Dis 2007;26:755-766.

9. Park SY, Lee SO, Choi SH, et al. Aspergillus galactomannan antigen assay in bronchoalveolar lavage fluid for diagnosis of invasive pulmonary aspergillosis. J Infect 2010;61:492-498.

10. Okuturlar Y, Ozkalemkas F, Ener B, et al. Serum galactomannan levels in the diagnosis of invasive aspergillosis. Korean J Intern Med 2015;30:899-905.

11. Kim SH, Kim MY, Hong SI, et al. Invasive pulmonary aspergillosis-mimicking tuberculosis. Clin Infect Dis 2015;61:9-17.

12. Kontoyiannis DP, Wessel VC, Bodey GP, Rolston KV. Zygomycosis in the 1990 s in a tertiary-care cancer center. Clin Infect Dis 2000;30:851-856.

13. Marr KA, Schlamm HT, Herbrecht R, et al. Combination antifungal therapy for invasive aspergillosis: a randomized trial. Ann Intern Med 2015;162:81-89.

14. Reed C, Bryant R, Ibrahim AS, et al. Combination polyene-caspofungin treatment of rhino-orbital-cerebral mucormycosis. Clin Infect Dis 2008;47:364-371.

15. Caillot D, Thiebaut A, Herbrecht R, et al. Liposomal amphotericin $\mathrm{B}$ in combination with caspofungin for invasive aspergillosis in patients with hematologic malignancies: a randomized pilot study (Combistrat trial). Cancer 2007;110:2740-2746.

16. Cornely OA, Maertens J, Bresnik M, Ullmann AJ, Ebrahimi R, Herbrecht R. Treatment outcome of invasive mould disease after sequential exposure to azoles and liposomal amphotericin B. J Antimicrob Chemother 2010;65:114-117.

17. Maertens JA, Raad II, Marr KA, et al. Isavuconazole versus voriconazole for primary treatment of invasive mould disease caused by Aspergillus and other filamentous fungi (SECURE): a phase 3, randomised-controlled, non-inferiority trial. Lancet 2016;387:760-769.

18. Walsh TJ, Raad I, Patterson TF, et al. Treatment of invasive aspergillosis with posaconazole in patients who are 
refractory to or intolerant of conventional therapy: an externally controlled trial. Clin Infect Dis 2007;44:2-12.

19. Pascual A, Calandra T, Bolay S, Buclin T, Bille J, Marchetti O. Voriconazole therapeutic drug monitoring in patients with invasive mycoses improves efficacy and safety outcomes. Clin Infect Dis 2008;46:201-211.

2o. Miyakis S, van Hal SJ, Ray J, Marriott D. Voriconazole concentrations and outcome of invasive fungal infections. Clin Microbiol Infect 2010;16:927-933.

21. Lee YJ, Lee SO, Choi SH, et al. Initial voriconazole trough blood levels and clinical outcomes of invasive aspergillosis in patients with hematologic malignancies. Med Mycol 2013;51:324-330.

22. Park WB, Kim NH, Kim KH, et al. The effect of therapeutic drug monitoring on safety and efficacy of voriconazole in invasive fungal infections: a randomized controlled trial. Clin Infect Dis 2012;55:1080-1087.

23. Abidi MZ, D’Souza A, Kuppalli K, Ledeboer N, Hari P. CY$\mathrm{P}_{2} \mathrm{C}_{1}{ }^{\star}{ }_{17}$ genetic polymorphism: an uncommon cause of voriconazole treatment failure. Diagn Microbiol Infect Dis 2015;83:46-48.

24. Jung J, Kim MY, Lee HJ, et al. Comparison of computed tomographic findings in pulmonary mucormycosis and invasive pulmonary aspergillosis. Clin Microbiol Infect 2015;21:684.

25. Caillot D, Latrabe V, Thiebaut A, et al. Computer tomography in pulmonary invasive aspergillosis in hematological patients with neutropenia: an useful tool for diagnosis and assessment of outcome in clinical trials. Eur J Radiol 2010;74:e172-e175.

26. Miceli MH, Maertens J, Buve K, et al. Immune reconstitution inflammatory syndrome in cancer patients with pulmonary aspergillosis recovering from neutropenia: proof of principle, description, and clinical and research implications. Cancer 2007;110:112-120.

27. Jung J, Hong HL, Lee SO, et al. Immune reconstitution inflammatory syndrome in neutropenic patients with invasive pulmonary aspergillosis. J Infect 2015;70:659-667.

28. Simitsopoulou M, Roilides E, Paliogianni F, et al. Im- munomodulatory effects of voriconazole on monocytes challenged with Aspergillus fumigatus: differential role of Toll-like receptors. Antimicrob Agents Chemother 2008;52:3301-3306.

29. Arendrup MC. Update on antifungal resistance in Aspergillus and Candida. Clin Microbiol Infect 2014;20 Suppl 6:42-48.

30. Verweij PE, Chowdhary A, Melchers WJ, Meis JF. Azole resistance in Aspergillus fumigatus: can we retain the clinical use of mold-active antifungal azoles? Clin Infect Dis 2016;62:362-368.

31. Howard SJ, Cerar D, Anderson MJ, Albarrag A, et al. Frequency and evolution of azole resistance in Aspergillus fumigatus associated with treatment failure. Emerg Infect Dis 2009;15:1068-1076.

32. van der Linden JW, Snelders E, Kampinga GA, et al. Clinical implications of azole resistance in Aspergillus fumigatus, The Netherlands, 2007-2009. Emerg Infect Dis 2011;17:1846-1854.

33. Escribano P, Pelaez T, Munoz P, Bouza E, Guinea J. Is azole resistance in Aspergillus fumigatus a problem in Spain? Antimicrob Agents Chemother 2013;57:2815-2820.

34. Toyotome T, Fujiwara T, Kida H, Matsumoto M, Wada T, Komatsu R. Azole susceptibility in clinical and environmental isolates of Aspergillus fumigatus from eastern Hokkaido, Japan. J Infect Chemother 2016;22:648-650.

35. Lepak AJ, Marchillo K, VanHecker J, Andes DR. Impact of in vivo triazole and echinocandin combination therapy for invasive pulmonary aspergillosis: enhanced efficacy against Cyp51 mutant isolates. Antimicrob Agents Chemother 2013;57:5438-5447.

36. van der Linden JW, Camps SM, Kampinga GA, et al. Aspergillosis due to voriconazole highly resistant Aspergillus fumigatus and recovery of genetically related resistant isolates from domiciles. Clin Infect Dis 2013;57:513-520.

37. Caillot D, Mannone L, Cuisenier B, Couaillier JF. Role of early diagnosis and aggressive surgery in the management of invasive pulmonary aspergillosis in neutropenic patients. Clin Microbiol Infect 2001;7 Suppl 2:54-61. 